\title{
Does Increased Arterial Stiffness Increase the Risk for Postural Hypotension?
}

\author{
David Sengstock, MD, MS; Peter V. Vaitkevicius, MD; Mark A. Supiano, MD \\ From the Department of Internal Medicine, Division of Geriatric Medicine, University of Michigan Health System, Ann Arbor, \\ Ml; and the Geriatric Research, Education, and Clinical Center, Veterans Affairs Health Care System, Ann Arbor, MI \\ Address for correspondence: David Sengstock, MD, MS, University of Michigan Ann Arbor Department of Veterans \\ Affairs Health System, Geriatric Research, Education, and Clinical Center (11G), 2215 Fuller Road, Ann Arbor, Ml 48105 \\ E-mail: dsmd@umich.edu \\ Manuscript received March 1, 2005; revised April 26, 2005; accepted May 4, 2005
}

This study tests the hypothesis that increased arterial stiffness is associated with postural hypotension in older adults. Aortic pulse wave velocity and postural blood pressure $(B P)$ response were assessed in 49 nondiabetic community-dwelling normotensive $(n=27)$ and hypertensive $(n=22)$ older adults (mean age $\pm S D, 71 \pm 6.7$ years) who were not receiving vasoactive medications. During the 5-minute period of upright posture, 13 subjects had no change or a postural increase in systolic $B P(S B P)(+10.6 \pm 14.6 \mathrm{~mm} \mathrm{Hg}), 27$ had a postural decrease of $<20 \mathrm{~mm} \mathrm{Hg}(-9.3 \pm 4.2 \mathrm{~mm} \mathrm{Hg})$, and nine had a postural decrease of $>20 \mathrm{~mm} \mathrm{Hg}(-29.1 \pm 8.1 \mathrm{~mm} \mathrm{Hg})$. Contrary to the proposed hypothesis, pulse wave velocity was significantly greater in subjects with a postural increase in SBP than in those with a postural decrease in $\mathrm{SBP}<20 \mathrm{~mm} \mathrm{Hg}(10.2 \pm 0.68 \mathrm{~m} / \mathrm{sec} v \mathrm{~s} .8 .3 \pm 0.37 \mathrm{~m} / \mathrm{sec} ; \mathrm{p}=0.03)$ and tended to be greater than in those with a postural decrease in $S B P>20 \mathrm{~mm} \mathrm{Hg}(10.2 \pm 0.68$ $\mathrm{m} / \mathrm{s}$ vs. $8.5 \pm 0.73 \mathrm{~m} / \mathrm{sec} ; \mathrm{p}=0.11)$. Higher pulse wave velocity was associated with a more positive postural SBP response at 1 minute $(\mathrm{r}=0.42 ; \mathrm{p}=0.024), 3$ minutes $(\mathrm{r}=0.38 ; \mathrm{p}=0.007)$, and 5 minutes $(\mathrm{r}=0.45 ; \mathrm{p}=0.001)$. This study does not support a relationship between arterial stiffness and a postural decrease in BP among healthy older adults; other age-related factors regulating $\mathrm{BP}$ homeostasis likely play a greater role. (AJGC. 2005;14:224-229) ${ }^{\circ} 2005$ Le Jacq Ltd.

$\mathrm{P}^{2}$ ostural hypotension has been reported to affect between $6 \%$ and $30 \%$ of communitydwelling individuals older than 65 years of age. ${ }^{1}$ During postural stress, a complex system of cardiovascular responses is required to maintain blood pressure (BP). These responses include the triggering of arterial baroreceptors and the sympathetic nervous system as well as an appropriate end-organ response, including vasoconstriction and an increase in heart rate. ${ }^{2}$ Older individuals are at risk for postural hypotension due to agerelated changes in both the afferent and efferent limbs of the baroreflex arc, ${ }^{3}$ heightened sympathetic nervous system activity, ${ }^{4}$ and impaired $\alpha$-adrenergic ${ }^{5}$ and $\beta$-adrenergic ${ }^{6}$ receptor responsiveness of target organs. Clinical studies have suggested that postural hypotension increases the risk

ID: 4503 for falls ${ }^{7}$ and stroke ${ }^{8}$ and is an independent predictor of mortality. ${ }^{9}$ Although risk factors such as age, diabetes, hypertension, and vasoactive medication use have been identified, the pathogenesis of this disorder remains incompletely understood. ${ }^{10-12}$

Age, diabetes, and hypertension are also closely associated with increased central artery stiffness. ${ }^{13}$ Recently, a study reported an association between arterial stiffness and orthostatic hypotension. ${ }^{14}$ The authors suggest that treatments aimed at decreasing arterial stiffness may lead to improvement in postural BP response. However, the study population consisted of hospitalized older patients with multiple comorbidities, including ischemic heart disease, atrial fibrillation, and diabetes, and at least one third of these patients were using vasoactive medications at the time of measurement. These comorbidities and their treatments have been independently associated with 
arterial stiffness, which may have confounded the reported relationship. Therefore, to avoid these confounding limitations, healthy older ambulatory normotensive and hypertensive individuals who were not actively treated with vasoactive medications were recruited for this study. This study tests the hypothesis that increased arterial stiffness is associated with the postural fall in BP in healthy and moderately hypertensive older adults.

\section{METHODS}

Subject Selection. Normotensive and hypertensive men and women in good general health aged 60-90 years were recruited for this cross-sectional study. Volunteers with a known history of symptomatic cardiac disease, arrhythmia, diabetes, or kidney disease were excluded. Volunteers with a serum creatinine $>1.5 \mathrm{mg} / \mathrm{dL}$ or fasting glucose $\geq 126 \mathrm{mg} / \mathrm{dL}$ were also excluded. All subjects had a normal sinus rhythm on electrocardiogram (ECG) at the time of the study. Volunteers taking antihypertensive medication were asked to complete a 4-week withdrawal period. If their BP exceeded 180/110 $\mathrm{mm} \mathrm{Hg}$, they were excluded from the study and their medication was restarted. Volunteers using other substances that could alter BP, e.g., tobacco or decongestants, were also excluded. Volunteers signed an informed consent form approved by the University of Michigan Institutional Review Board.

Thirty-eight normotensive volunteers, free of vasoactive medications, were enrolled. Eleven hypertensive volunteers completed the 4-week antihypertensive medication withdrawal and were also enrolled. Subjects were studied by a single operator in the morning following an overnight fast free of caffeine products.

Arterial Stiffness. Aortic stiffness was assessed by measuring the carotid-femoral pulse wave velocity (PWV)..$^{15}$ Subjects were placed in a supine position and ECG leads were positioned to continuously record heart rate. Systolic BP (SBP) and diastolic BP (DBP) were measured with a manual sphygmomanometer. Subsequently, the location of the maximal impulse of the right common carotid and right common femoral arterial pulses were marked, as was the midpoint of the manubrium. To approximate the length of the descending aorta, the distance from the midpoint of the manubrium to the maximal pulse of the right carotid artery was subtracted from the distance from the midpoint of the manubrium to the maximal pulse of the right femoral artery $\left(l_{\text {aorta }}[\mathrm{mm}]\right)$.
A hand-held high-fidelity tonometer (SPC-301; Millar Instruments, Houston, TX) was placed over the maximal impulse of the carotid artery to achieve a pressure wave contour with a consistent baseline, contour, and amplitude. A 20-second time span of these carotid pulse contours was recorded (AtCor version 7.0; AtCor Medical Inc. (USA), Chicago, IL). The average time $\left(\mathrm{t}_{\mathrm{c}}[\mathrm{ms}]\right)$ between each R wave on the ECG and the foot of the corresponding carotid pressure waveform was calculated. Similarly, the tonometer was placed over the maximal impulse of the right common femoral artery to calculate $\mathrm{t}_{\mathrm{f}}(\mathrm{ms})$. $\mathrm{PWV}(\mathrm{m} / \mathrm{sec})$ was then calculated by the equation PWV $=1_{\text {aorta }} /\left(t_{\mathrm{f}}-\mathrm{t}_{\mathrm{c}}\right)$. A measurement was excluded if the pressure contour was of poor quality or if a significant difference $(>15 \%)$ in heart rate was found between the carotid and femoral measurements. Four PWV measurements were recorded for each subject. A subject's PWV was the average of the technically acceptable measurements.

To estimate intraoperator reproducibility of PWV, one operator completed serial measurements in a subset of 18 volunteers on three study visits separated by 1 week. To estimate interoperator reliability, two operators independently measured PWV in a subset of 22 volunteers during a single occasion. The intraoperator coefficient of variation for PWV measures obtained during three separate visits was $5.8 \% \pm 3.6 \%$. The interoperator coefficient of variation for PWV on the same visit was $5.1 \% \pm 3.7 \%$.

Upright Posture Protocol. Following 30 minutes of supine rest while PWV measurements were performed, baseline SBP and DBP were measured with a manual sphygmomanometer. Supine BP measurements were repeated until three consecutive measurements were within $\pm 2 \mathrm{~mm} \mathrm{Hg}$. These consecutive measurements were averaged. Subjects were then brought to a standing position in a standardized fashion in less than 10 seconds. BP and heart rate were recorded at 1,3 , and 5 minutes. The postural change in BP was calculated as the difference between the average supine $\mathrm{BP}$ and the pressures at 1, 3, and 5 minutes of standing. The lowest of the three values was used to classify subjects as having no fall in SBP, a fall $<20 \mathrm{~mm} \mathrm{Hg}$, or a fall $\geq 20 \mathrm{~mm} \mathrm{Hg}$.

Statistical Analysis. A sample size of $\mathrm{N}=47$ was targeted, providing $80 \%$ power to detect that PWV explains $15 \%$ of the variation in postural BP change. All analyses were made using SAS version 


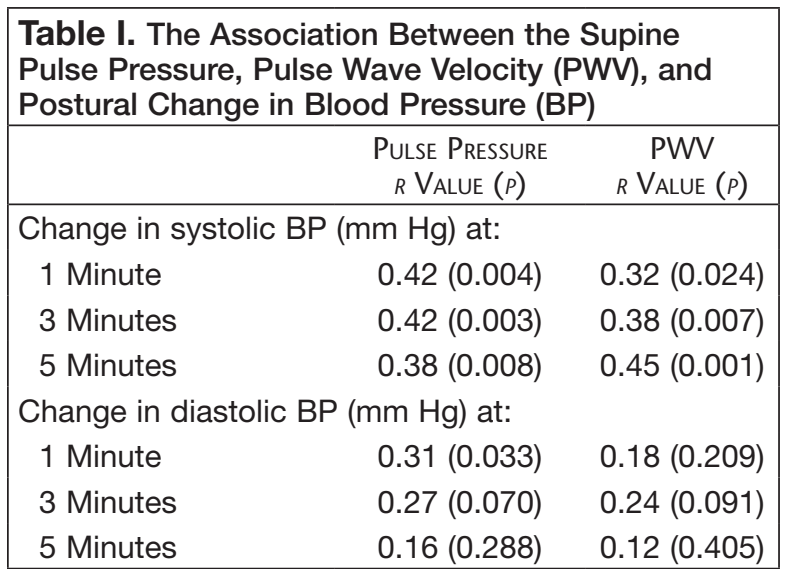

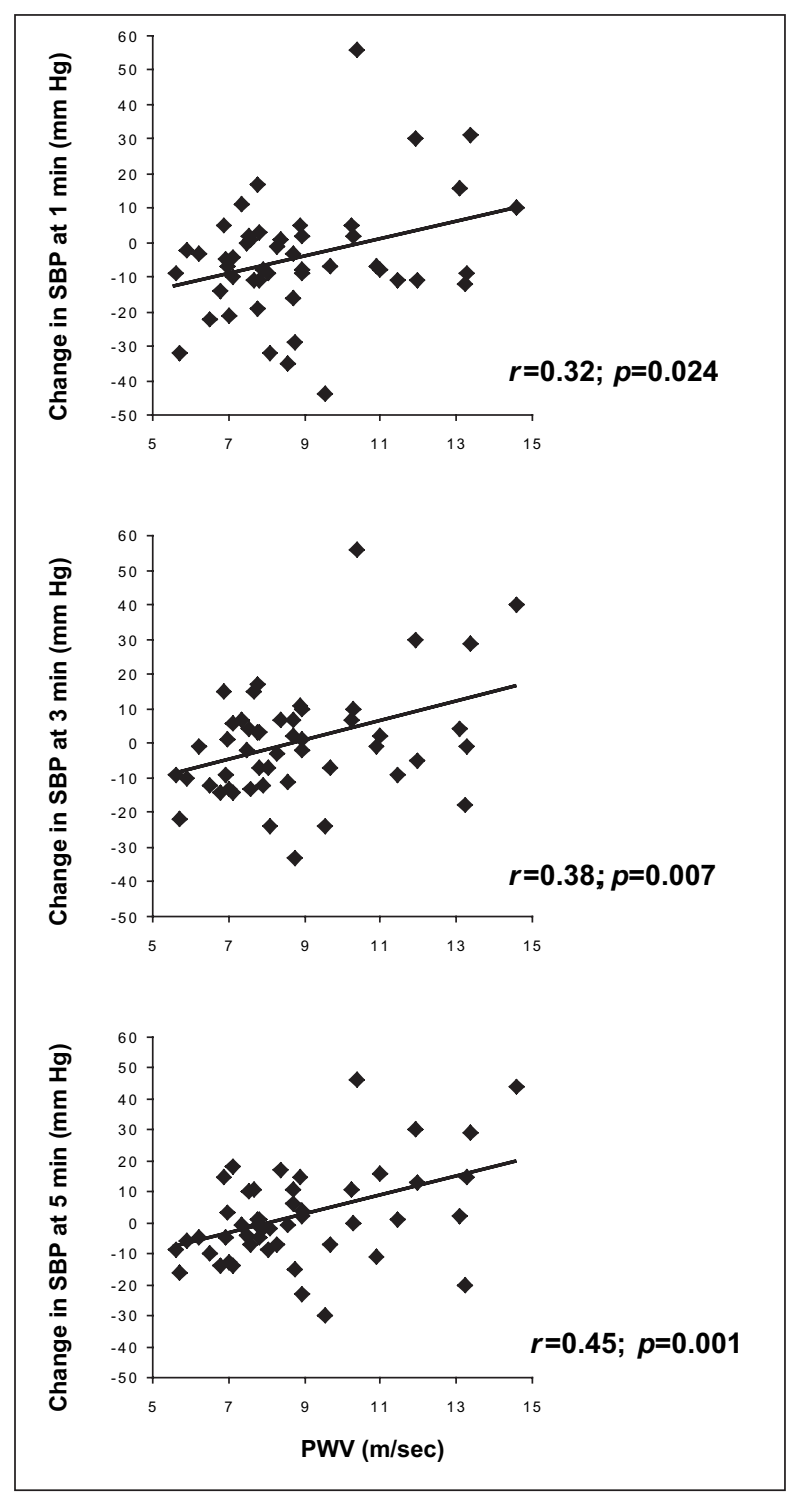

Figure 1. Associations between pulse wave velocity (PWV) and postural systolic blood pressure (SBP) response at 1,3 , and 5 minutes
8.0 (SAS Institute, Inc., Cary, NC). Continuous variables are expressed as means $\pm \mathrm{SD}$. Categoric variables are expressed as percentages. A Student $t$ test was used to compare group means. Subjects were categorized by their postural BP change during the 5-minute stand as: 1) no change or a postural increase in SBP; 2) a postural decrease between 1 and $20 \mathrm{~mm} \mathrm{Hg}$; or 3) a postural decrease greater than $20 \mathrm{~mm} \mathrm{Hg}$. A one-way analysis of variance was used to analyze the association between baseline characteristics and these categories of postural $\mathrm{BP}$ change. Linear regression was performed to analyze the associations between PWV and the postural changes in SBP and DBP, as well as with previously reported predictors of postural pressure changes. Multiple linear regressions were performed to assess the independence of these associations.

\section{RESULTS}

The average age of the subjects studied (22 men and 27 women) was $71 \pm 6.7$ (range 60-87) years. The mean body mass index (BMI) was $25.3 \pm 3.6$ (range, 18.4-36.3) kg/m². Eleven subjects (22\%) had a history of drug treatment for hypertension (mean SBP, $154 \pm 14.5 \mathrm{~mm} \mathrm{Hg}$ ); 13 subjects (27\%) with no history of hypertension had resting supine SBP $\geq 140 \mathrm{~mm} \mathrm{Hg}$ (mean SBP, $150 \pm 8.5$ $\mathrm{mm} \mathrm{Hg}$ ). For the entire sample, the mean resting supine SBP was $139 \pm 19$ (range 96-180) mm Hg; the mean resting supine DBP was $69.8 \pm 9.5$ (range 41-86) $\mathrm{mm} \mathrm{Hg}$. The mean resting supine heart rate was $59.9 \pm 7.6$ (range $42-77$ ) bpm.

The mean PWV and pulse pressure (PP) were $8.8 \pm 2.2 \mathrm{~m} / \mathrm{secec}$ (range $5.6-14.6 \mathrm{~m} / \mathrm{sec}$ ) and $68.7 \pm 16 \mathrm{~mm} \mathrm{Hg}$ (range $34-102 \mathrm{~mm} \mathrm{Hg}$ ) respectively. In univariate analysis, PWV correlated with resting SBP $(r=0.72 ; p<0.001)$, PP $(r=0.63 ; p<0.001)$, DBP $(r=0.29 ; p<0.044)$, heart rate $(r=0.47 ; p<0.001)$, age $(r=0.32 ; p=0.024)$, and BMI $(r=0.33 ; p=0.022)$. In multiple regression, PWV was independently associated with SBP $(p<0.001)$, heart rate $(p=0.003)$, BMI $(p=0.016)$, and age $(p=0.026)$; this model explained $67 \%$ of the variance in PWV.

No significant relationship was found between age or resting supine SBP and the postural change in SBP or DBP during the 5 -minute stand $(p>0.2$ for all time points). The associations between PWV or PP and the change in SBP and DBP at 1, 3, and 5 minutes are shown in Table I. A direct association was seen between PWV and the postural change in SBP at all three time points (Figure 1). This relationship remained significant $(p<0.05$ for 
Table II. Baseline Characteristics* by Postural Change in Systolic Blood Pressure (SBP) During the 5-Minute Stand

\begin{tabular}{|c|c|c|c|c|}
\hline & $\begin{array}{c}\uparrow \mathrm{SBP} \geq 0 \mathrm{MM} \mathrm{HG} \\
(\mathrm{N}=13)\end{array}$ & $\begin{array}{c}\downarrow \text { SBP } 0-20 \mathrm{MM} \mathrm{HG} \\
(\mathrm{N}=27)\end{array}$ & $\begin{array}{c}\downarrow \mathrm{SBP}>20 \mathrm{MM} \mathrm{HC} \\
(\mathrm{N}=9)\end{array}$ & $\begin{array}{l}\text { TREND } \\
p \text { VALUE } \\
\end{array}$ \\
\hline Age (yr) & $71.8 \pm 5.7$ & $69.3 \pm 6.9$ & $75.7 \pm 5.8$ & 0.30 \\
\hline Males & $4(31)$ & $12(40)$ & $7(78)$ & 0.04 \\
\hline Body mass index $\left(\mathrm{kg} / \mathrm{m}^{2}\right)$ & $26.8 \pm 5.0$ & $24.6 \pm 3.0$ & $25.3 \pm 1.9$ & 0.24 \\
\hline History of hypertension & $5(38)$ & $12(44)$ & $3(33)$ & 0.88 \\
\hline Supine SBP (mm Hg) & $139 \pm 20.3$ & $140 \pm 19$ & $132 \pm 20$ & 0.45 \\
\hline Supine diastolic BP $(\mathrm{mm} \mathrm{Hg})$ & $65 \pm 11$ & $72 \pm 8.2$ & $70.1 \pm 9.8$ & 0.18 \\
\hline Supine heart rate (bpm) & $62 \pm 5.3$ & $59.9 \pm 8.7$ & $56.7 \pm 6.5$ & 0.11 \\
\hline
\end{tabular}

all time points) when controlling for sex, resting heart rate, or age. Similarly, a direct relationship was seen between PP and the postural change in SBP at 1, 3, and 5 minutes; this association also remained statistically significant $(p<0.03$ for all comparisons) when controlling for sex, resting heart rate, or age. A direct relationship was also found between PP and the change in DBP at 1 and 3 minutes, although the latter time point did not reach statistical significance.

The postural change in SBP was defined as the difference between the supine SBP and the lowest of the three SBP measurements during the 5-minute stand. Thirteen subjects had no change or a postural increase in SBP $(+10.6 \pm 14.6 \mathrm{~mm} \mathrm{Hg}$; range $0-46$ $\mathrm{mm} \mathrm{Hg}) ; 27$ subjects had a postural decrease between 1 and $20 \mathrm{~mm} \mathrm{Hg}(-9.3 \pm 4.2 \mathrm{~mm} \mathrm{Hg}$; range -19 to $-1 \mathrm{~mm} \mathrm{Hg}$ ); nine subjects had a postural decrease $>20 \mathrm{~mm} \mathrm{Hg}(-29.1 \pm 8.1 \mathrm{~mm} \mathrm{Hg}$; range -44 to -20 $\mathrm{mm} \mathrm{Hg})$. Baseline characteristics by these three categories of postural BP response are shown in Table II. Men exhibited a significantly greater postural fall in BP than women $(-14 \pm 13 \mathrm{~mm} \mathrm{Hg}$ vs. $-2.4 \pm 16$ $\mathrm{mm} \mathrm{Hg} ; p=0.010)$. The postural change in SBP correlated with resting PP $(r=0.32 ; p<0.03)$, resting PWV $(r=0.34 ; p<0.02)$, marginally with resting DBP $(r=-0.25 ; p<0.08)$, but did not correlate with BMI, history of hypertension, resting SBP, or heart rate $(p>0.1$ for all). In stepwise multiple regression, the postural change in SBP was independently associated with resting PWV $(p<0.003)$ and resting DBP $(p<0.03)$ and tended to decrease in men $(p=0.06)$; this model explained $27 \%$ of the variance in PWV.

During the 5-minute stand, dizziness was reported by two of the $13(15 \%)$ individuals with no change or a postural increase in SBP, by seven of the 27 (26\%) individuals with a postural decrease between 1 and 20 $\mathrm{mm} \mathrm{Hg}$, and by three of the nine individuals (33\%) with an SBP fall $\geq 20 \mathrm{~mm} \mathrm{Hg}$. The average PWV was greater in subjects with no postural decrease in SBP than in those with a postural decrease $<20 \mathrm{~mm} \mathrm{Hg}$ $(10.2 \pm 2.4 \mathrm{~m} / \mathrm{sec}$ vs. $8.3 \pm 1.9 \mathrm{~m} / \mathrm{sec} ; p=0.03)$ (Figure 2). Similarly, the average PWV tended to be greater in subjects with no postural decrease in SBP than in those with a postural decrease $>20 \mathrm{~mm} \mathrm{Hg}(10.2 \pm 2.4$ $\mathrm{m} / \mathrm{sec}$ vs. $8.5 \pm 2.2 \mathrm{~m} / \mathrm{sec} ; p=0.11)$.

\section{DISCUSSION}

The results of this study fail to demonstrate an association between increased arterial stiffness and a postural decrease in SBP or DBP. The present study was performed in a population of healthy older normotensive and hypertensive adults who were not receiving vasoactive medications. This subject selection minimized the confounding effects of medications and comorbid medical conditions on the postural BP response and its relationship to arterial stiffness. Although individuals with a known history of orthostatic hypotension were excluded, nearly $20 \%$ of the subjects studied exhibited a fall in SBP of 20 $\mathrm{mm} \mathrm{Hg}$ during the 5-minute period of upright posture. When these individuals with orthostatic hypotension were compared with the group with no change or a postural increase in SBP, their PWV tended to be lower, not higher.

Recently, Boddaert et al. ${ }^{14}$ reported that among older individuals hospitalized for complications related to a fall, arterial stiffness was greater in individuals with orthostatic hypotension compared with those without orthostatic hypotension. It was proposed that stiff artery walls might restrict the mechanical stretch of arterial baroreceptors, thereby blunting the compensatory response to upright posture. A recent study of healthy and hypertensive older adults failed to demonstrate such an association between baroreflex sensitivity and arterial stiffness. ${ }^{16}$ Results from the present study do not support a postural BP fall-arterial stiffness relationship. 


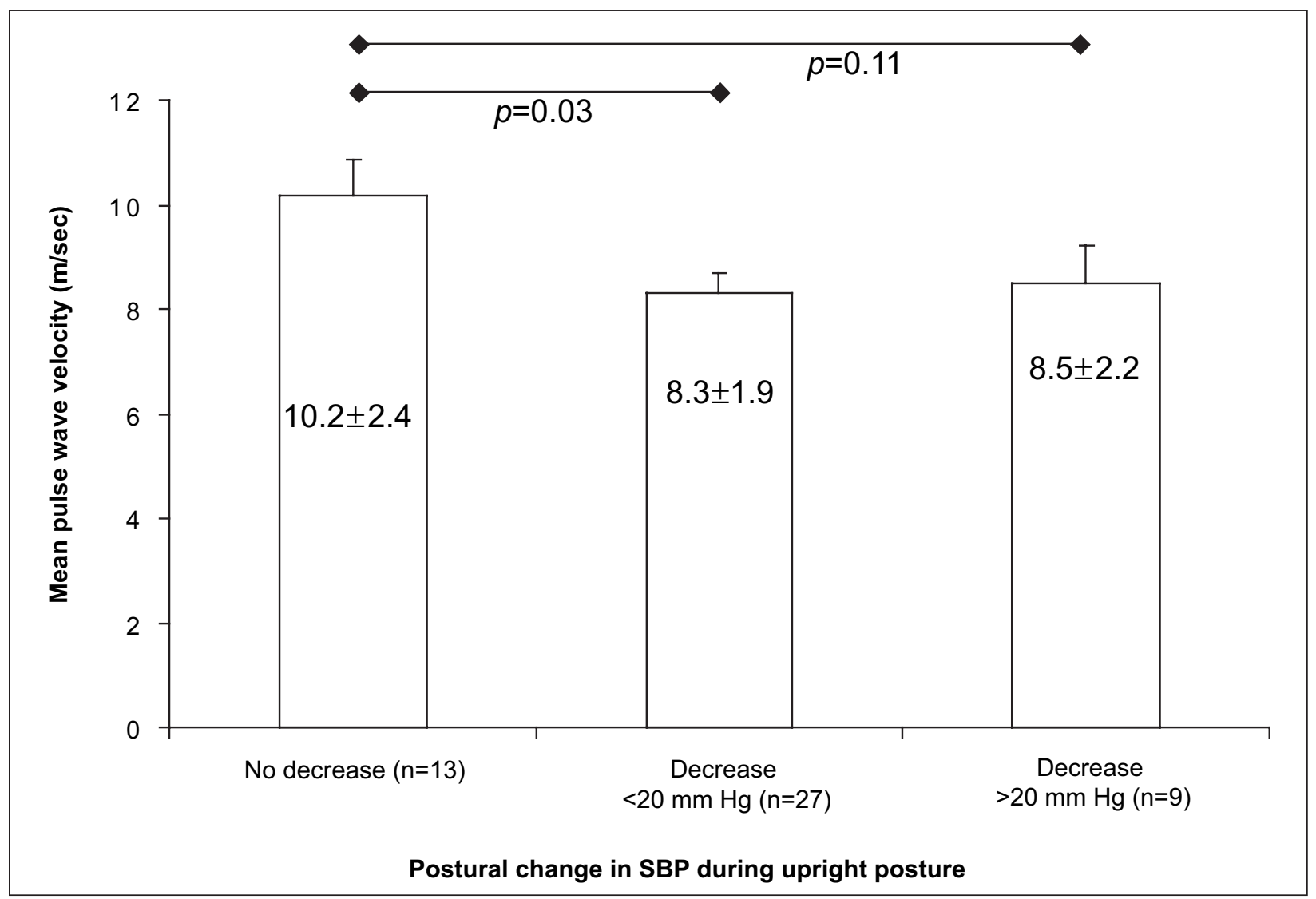

Figure 2. Pulse wave velocity grouped by the postural change in systolic blood pressure (SBP): no decrease, a decrease $<20 \mathrm{~mm} \mathrm{Hg}$, or a decrease $>20 \mathrm{~mm} \mathrm{Hg}$

The conflicting results between the present study and the Boddaert et al. study are likely due to critical differences in the study populations. First, the present study was performed in a healthy ambulatory population, while the previous study examined patients acutely hospitalized for complications related to a fall. Second, subjects in the present study were free of any vasoactive medications, while at least one third of the subjects in the previous study reported the active use of vasoactive medications including nitrates, calcium channel blockers, and angiotensin-converting enzyme inhibitors. Third, the subjects in the present study were normotensive and hypertensive older adults who were carefully screened to be in good general health; the previous study included individuals with ischemic heart disease, atrial fibrillation, heart failure, diabetes, and stroke. Furthermore, patients in the previous study who exhibited postural hypotension had a higher prevalence of cardiac disease and atrial fibrillation compared with those without hypotension. Studies have shown that individuals with cardiovascular disease have a diminished ejection time and heart rate response to postural stress. ${ }^{17-19}$ Given the findings of the present study, the relationship identified between orthostatic hypotension and arterial stiffness in the Boddaert et al. study was more likely due to these medical conditions and medications than the increase in arterial stiffness that occurs with aging.

Interestingly, a direct relationship was demonstrated between the severity of arterial stiffness and a postural increase in SBP. As shown in Figure 1, individuals with greater arterial stiffness were more inclined to increase their BP when challenged with a postural stress. Although it was not discussed, this finding was also reported in the Boddaert et al. study. ${ }^{14}$ During postural stress, cardiovascular mechanisms are employed to maintain SBP, primarily by increasing heart rate and stroke volume. One could hypothesize that when an increase in cardiac output is directed into a stiff arterial tree, a greater increase in SBP would occur than when a similar load is directed into a more compliant arterial tree. ${ }^{20}$ Although this speculation requires further study, the arterial stiffness-postural increase in SBP relationship does offer further evidence against the hypothesis that the age-related increase in arterial stiffness is associated with a greater risk for postural BP decline. 
It is important to note several limitations in this study. By design, the study did not include individuals with active medical conditions other than hypertension, nor were individuals on vasoactive medications included. Indeed, these conditions may have been responsible for the previous observation that arterial stiffness was higher in those with postural hypotension. The current study was also not designed to assess arterial stiffness in individuals with a history of falls or a history or symptoms of postural hypotension. The study design is also limited in part due to the welldefined day-to-day variability reported in postural BP response. ${ }^{21-23}$ Measurements in this study were taken on a single visit and, therefore, do not provide information about day-to-day postural BP variations that are likely to exist. However, given that a statistically significant relationship was observed between arterial stiffness and postural SBP increase, it seems unlikely that the day-to-day variability in postural BP response would lead to the opposite finding on a different day.

\section{CONCLUSION}

This study fails to identify an association between increased arterial stiffness and the postural decrease in SBP among healthy normotensive and moderately hypertensive older individuals. Other factors regulating BP homeostasis likely play a greater role than the age-related increase in arterial stiffness in maintaining BP during upright posture.

Acknowledgments: The authors acknowledge the important contributions of many individuals to this study: Kathy Jarvenpaa and the General Clinical Research Center (GCRC) nursing staff, Cathie Bloem, and Jeffrey Briesmiester for technical support. This work was supported in part by: the Department of Veterans Affairs Medical Research Service/Ann Arbor Geriatric Research Education and Clinical Center, Hartford/American Federation of Aging Research Academic Geriatric Fellowship Award, University of Michigan Institute of Gerontology "Multidisciplinary Research Training in Aging", Grant (T32 AG00114), University of Michigan Training Program in Clinical Research Award (1 K30 HL04108-02), and the University of Michigan General Clinical Research Center (M01-RR00042 and K24 AG00924 [MAS]).

\section{REFERENCES}

1 Mader SL, Josephson KR, Rubenstein LZ. Low prevalence of postural hypotension among community-dwelling elderly. JAMA. 1987;258:1511-1514.

2 Smit AA, Halliwill JR, Low PA, et al. Pathophysiological basis of orthostatic hypotension in autonomic failure. J Physiol. 1999;519(pt 1):1-10.

3 Gribbin B, Pickering TG, Sleight P, et al. Effect of age and high blood pressure on baroreflex sensitivity in man. Circ Res. 1971;29:424-431.
4 Supiano MA, Linares OA, Smith MJ, et al. Age-related differences in norepinephrine kinetics: effect of posture and sodium-restricted diet. Am J Physiol. 1990;259:E422-E431.

5 Hogikyan RV, Supiano MA. Arterial alpha-adrenergic responsiveness is decreased and SNS activity is increased in older humans. Am J Physiol. 1994;266:E717-E724.

6 Ford GA, Hoffman BB, Blaschke TF. Beta adrenergically mediated cardiac chronotropic and vascular smooth muscle responses during propranolol therapy and withdrawal in young and elderly persons. J Gerontol. 1992;47:M22-M26.

7 Tinetti ME, Williams TF, Mayewski R. Fall risk index for elderly patients based on number of chronic disabilities. Am J Med. 1986;80:429-434.

8 Eigenbrodt ML, Rose KM, Couper DJ, et al. Orthostatic hypotension as a risk factor for stroke: the atherosclerosis risk in communities (ARIC) study, 1987-1996. Stroke. 2000;31:2307-2313.

9 Masaki KH, Schatz IJ, Burchfiel CM, et al. Orthostatic hypotension predicts mortality in elderly men: the Honolulu Heart Program. Circulation. 1998;98:2290-2295.

10 Mader SL. Aging and postural hypotension. An update. J Am Geriatr Soc. 1989;37:129-137.

11 Lipsitz LA. Orthostatic hypotension in the elderly. $N$ Engl J Med. 1989;321:952-957.

$12 \mathrm{Wu}$ JS, Lu FH, Yang YC, et al. Postural hypotension and postural dizziness in patients with non-insulin-dependent diabetes. Arch Intern Med. 1999;159:1350-1356.

13 Mackey RH, Sutton-Tyrrell K, Vaitkevicius PV, et al. Correlates of aortic stiffness in elderly individuals: a subgroup of the Cardiovascular Health Study. Am J Hypertens. 2002;15:16-23.

14 Boddaert J, Tamim H, Verny M, et al. Arterial stiffness is associated with orthostatic hypotension in elderly subjects with history of falls. J Am Geriatr Soc. 2004;52:568-572.

15 Van Bortel LM, Duprez D, Starmans-Kool MJ, et al. Clinical applications of arterial stiffness, Task Force III: recommendations for user procedures. Am J Hypertens. 2002;15:445-452.

16 Mukai S, Gagnon M, Iloputaife I, et al. Effect of systolic blood pressure and carotid stiffness on baroreflex gain in elderly subjects. J Gerontol A Biol Sci Med Sci. 2003;58:626-630.

17 Jefrey FE, Fareeduddin K, Abelmann WH. Increased tolerance of patients with circulatory congestion to orthostatic stress. Am J Med Sci. 1970;259:323-332.

18 Abelmann WH, Fareeduddin K. Increased tolerance of orthostatic stress in patients with heart disease. $A m ~ J$ Cardiol. 1969;23:354-363.

19 ZambranoSS, Spodick DH. Comparative responses to orthostatic stress in normal and abnormal subjects. Evaluation by impedance cardiography. Chest. 1974;65:394-396.

20 Nichols WW, O'Rourke MF, eds. McDonald's Blood Flow in Arteries: Theoretic, Experimental, and Clinical Principles. 3rd ed. London, England: Edward Arnold; 1990.

21 Belmin J, Abderrhamane M, Medjahed S, et al. Variability of blood pressure response to orthostatism and reproducibility of the diagnosis of orthostatic hypotension in elderly subjects. J Gerontol A Biol Sci Med Sci. 2000;55: M667-M671.

22 Weiss A, Grossman E, Beloosesky Y, et al. Orthostatic hypotension in acute geriatric ward: is it a consistent finding? Arch Intern Med. 2002;162:2369-2374.

23 Ward C, Kenny RA. Reproducibility of orthostatic hypotension in symptomatic elderly. Am J Med. 1996;100:418-422. 